\title{
Inferior vena cava filter insertion through the popliteal vein: enabling the percutaneous endovenous intervention of deep vein thrombosis with a single venous access approach in a single session
}

\author{
Hyoung Ook Kim \\ Jae Kyu Kim \\ Jin Gyoon Park \\ Nam Yeol Yim \\ Yang Jun Kang \\ Hye Doo Jung
}

From the Department of Radiology (J.K.K. $₫ k j k r a d @$ chonnam.ac.kr), Chonnam National University Hospital, Dong-gu, Gwangju, Korea.

Received 9 August 2015; revision requested 21 September 2015; last revision received 1 February 2016; accepted 27 February 2016.

Published online 22 August 2016. DOI 10.5152/dir.2016.15347

\begin{abstract}
PURPOSE
We aimed to evaluate the efficiency of placing an inferior vena cava (IVC) filter through the same popliteal vein access site used for percutaneous endovenous intervention in patients with extensive lower extremity deep vein thrombosis.

\section{METHODS}

This retrospective study included 21 patients who underwent IVC filter insertion through the popliteal vein over a three-year period. Patient medical records were reviewed for the location of the deep vein thrombosis, result of filter removal, and total number of endovascular procedures needed for filter insertion and recanalization of the lower extremity venous system. Follow-up lower extremity computed tomography (CT) venography was also reviewed in each patient to assess the degree of filter tilt in the IVC.
\end{abstract}

\section{RESULTS}

All patients had extensive lower extremity deep vein thrombosis involving the iliac vein and/or femoral vein. Seventeen patients showed deep vein thrombosis of the calf veins. In all patients, IVC filter insertion and the recanalization procedure were performed during a single procedure through the single popliteal vein access site. In the 17 patients undergoing follow-up CT, the mean tilt angle of the filter was $7.14^{\circ} \pm 4.48^{\circ}$ in the coronal plane and $8.77^{\circ} \pm 5.49^{\circ}$ in the sagittal plane. Filter retrieval was successful in 16 of 17 patients $(94.1 \%)$ in whom filter retrieval was attempted.

\section{CONCLUSION}

Transpopliteal IVC filter insertion is an efficient technique that results in low rates of significant filter tilt and enables a single session procedure using a single venous access site for filter insertion and percutaneous endovenous intervention.
$\mathrm{R}$ etrievable inferior vena cava (IVC) filters are effective for reducing the risk of pulmonary embolism (PE) from lower extremity deep vein thrombosis (DVT) (1). In addition, prophylactic IVC filter insertion has been proposed as a strategy to prevent PE during percutaneous endovenous intervention (PEVI) such as catheter-directed thrombolysis and mechanical thrombectomy of proximal DVT (2). PEVI is being increasingly used as a powerful tool in the management of acute proximal DVT. Instrumentation of extensive fresh thrombus during $\mathrm{PEVI}$ is potentially associated with iatrogenic $\mathrm{PE}$, and the placement of a retrievable filter in the IVC during thrombolytic therapy, angioplasty, and stent placement can prevent silent and symptomatic $\mathrm{PE}$ in patients with thrombosis of the iliocaval segment (3).

Placement of an IVC filter is commonly accomplished using the right femoral or right internal jugular vein approach with the patient in the supine position. However, the preferential venous access site for PEVI is the popliteal vein with the patient in the prone position. Therefore, prophylactic IVC filter insertion and PEVI usually require a minimum of two venous access sites, which increases the potential for hemorrhage and patient discomfort.

This study examines the efficiency of IVC filter insertion through a popliteal vein approach in patients with extensive lower extremity DVT.

\section{Methods}

\section{Study population}

This retrospective study received institutional review board approval. We retrospectively analyzed the medical records of 21 patients who underwent IVC filter insertion via popliteal 
venous access between January 2012 and July 2015. Thirteen patients were women and eight patients were men. The mean age was 61.7 years (range, 30-86 years). Data describing the symptom duration, predisposing factors for DVT, location of DVT, D-dimer levels within three days before the procedure, filter duration data, and amount of contrast agent (iohexol, Omnihexol 300, Korea United Pharm Co.) used during IVC filter insertion and PEVI were collected from the medical records of each patient, and lower extremity computed tomography (CT) venography data were obtained. The rethrombosis rate was evaluated in patients who underwent follow-up CT venography or Doppler sonography after IVC filter insertion and PEVI.

\section{Inferior vena cava filter insertion}

Anticoagulation management was performed in 18 of 21 patients before IVC filter insertion. Subcutaneous low-molecular-weight heparin $(n=12)$, oral rivaroxaban $(n=7)$, oral warfarin $(n=6)$ and intravenous unfractionated heparin $(n=5)$ were used before the procedure.

In all patients, a popliteal vein approach in the leg with the venous thrombosis was attempted. The ipsilateral popliteal vein puncture was done under ultrasonography guidance, with a micro-puncture set employing a 21-gauge needle (Cook Medical), and was followed by the insertion of an $8 \mathrm{~F}$ angiography introducer sheath (Radifocus, Terumo).

A $5 \mathrm{~F}$ headhunter type angiographic catheter (Boston Scientific) was then introduced

\section{Main points}

Prophylactic inferior vena cava filter (IVC) placement has been proposed as a method to decrease the risk of pulmonary embolism when performing percutaneous endovenous intervention (PEVI) for deep vein thrombosis.

Usually, IVC filter insertion and PEVI require two separate procedures performed on different days because of the need for different access sites.

- IVC filter insertion through the ipsilateral popliteal vein is an efficient procedure that can be performed in conjunction with PEVI in a single session through a single access site.

In this study, the filter was successfully placed in all patients through the popliteal vein without procedural or post-procedure complications. Furthermore, this method was associated with a low occurrence of significant filter tilt. and passed through the venous segment containing the thrombosis. After the angiographic catheter was entered into the IVC lumen, a double-basket shaped retrievable IVC filter equipped with a $90 \mathrm{~cm}$ length introducer set (OptEase filter, Cordis) was introduced along a stiff guidewire (Cook Medical) and deployed in the infrarenal IVC under fluoroscopic guidance.

\section{Percutaneous endovenous intervention}

After IVC filter insertion, endovascular treatment, including aspiration thrombectomy or thrombolysis, was performed for iliofemoral vein thromboses. For thrombolysis, infusion was performed using a multi-sideport infusion catheter (Multi-Sideport catheter infusion set, Cook Medical). Before thrombolytic therapy, venography was once again performed to confirm the position of the catheter and evaluate the IVC thrombus. The catheter was placed within the thrombosed venous segment and urokinase (Green Cross) was administered via a continuous infusion (100 $000 \mathrm{IU} / \mathrm{h}$ ). Patients received systemic anticoagulation with a 3000 IU bolus of intravenous heparin and continuous infusion (3000 IU/h) of intravenous heparin via the side arm of the vascular sheath. The status of lysis was monitored using venography at 30 min intervals. If only partial lysis was achieved, the infusion catheter was repositioned within the residual thrombus and the infusion was continued. The start and end times of thrombolysis and the total amount of urokinase administered were recorded. Aspiration thrombectomy was performed to clear softening thrombi. Aspiration thrombectomy was performed in a forward-and-backward manner using an $8 \mathrm{~F}$ guiding catheter (Guider soft tip XF; Boston Scientific) while maintaining negative pressure with a 20 or $50 \mathrm{~mL}$ syringe. To prevent body volume loss during aspiration thrombectomy, the same amount of heparinized saline was infused manually via the sheath side arm. The start and end times of aspiration thrombectomy were recorded. For any remaining flow-limiting lesions after thrombolysis or thrombectomy, adjuvant endovascular treatments such as balloon angioplasty or venous stenting were performed to completely restore venous flow. Initial dilatation for reconstruction of the iliac vein was achieved with an 8 to $12 \mathrm{~mm}$ diameter balloon catheter (Boston Scientific). Stents were placed over a guidewire into the iliac vein and were typically $10-14 \mathrm{~mm}$ in di- ameter and 6-8 cm in length. Smart stents (Cordis) and Protégé stents (ev3) were commonly used to reconstruct the iliac vein.

Filter removal was attempted through the right common femoral vein after endovascular treatment of the DVT. A loop snare device (ev3) was used for filter retrieval.

\section{Measuring filter tilt}

Filter tilt was defined as the angle between the long axis of the filter and the IVC wall. The long axis of the IVC filter is a virtual line between the cranial apex marker and the caudal hook. The filter tilt measurement was performed by three-dimensional rendering software (Aquarius iNtuition viewer, Terarecon). After loading the follow-up CT venography image data using this software, multiplanar reformatted images, including the coronal and sagittal planes, were obtained. With this reconstructed image data, the filter tilt was measured in the coronal and sagittal planes (Fig. 1). We also measured the filter tilt of 13 patients who underwent IVC filter insertion via contralateral femoral access between March 2012 and July 2015 to compare the difference in the degree of filter tilt between ipsilateral popliteal and contralateral femoral access.

\section{Statistical analysis}

To evaluate the efficacy of the transpopliteal venous IVC filter insertion, we analyzed the degree of filter tilt in each patient. According to the Society of Interventional Radiology definition (4), significant filter tilt considered as an insertion problem was defined as a filter tilt $\geq 15^{\circ}$ from the IVC axis as seen in the coronal or sagittal plane. The success of filter removal was also evaluated to assess the ease of filter removal. A paired t-test was performed to compare the difference in filter tilt degree between the coronal and sagittal planes. An independent samples t-test was performed to compare the difference in filter degree between the ipsilateral popliteal and contralateral femoral access sites. The level of significance was set as $P<0.01$ for the statistical analyses. The Statistical Package for the Social Sciences (SPSS 21.0 for windows, IBM Corp.) was used to perform the statistical analyses.

\section{Results}

The median DVT symptom duration for the enrolled patients was three days (range, 0.2-14 days) (Table). Seventeen patients showed thrombosis of the distal veins, such 

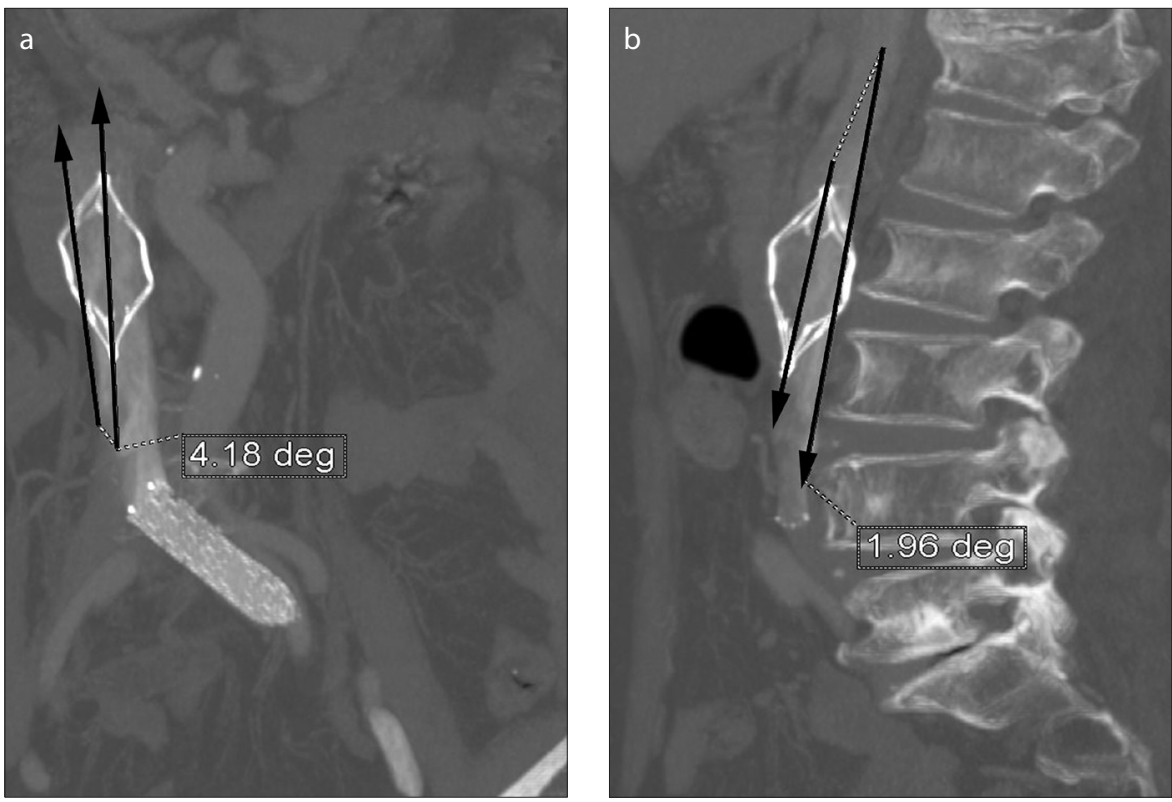

Figure 1. a, b. Measurement of filter tilt. Reconstructed coronal computed tomography (CT) image (a) shows the axis of the inferior vena cava (IVC) filter, with a tilt angle of $4.18^{\circ}$. Reconstructed sagittal CT image (b) shows the axis of the IVC filter with a tilt angle of $1.96^{\circ}$. Arrows in (a) and (b) indicate angle created by the axis of the filter with respect to the IVC.
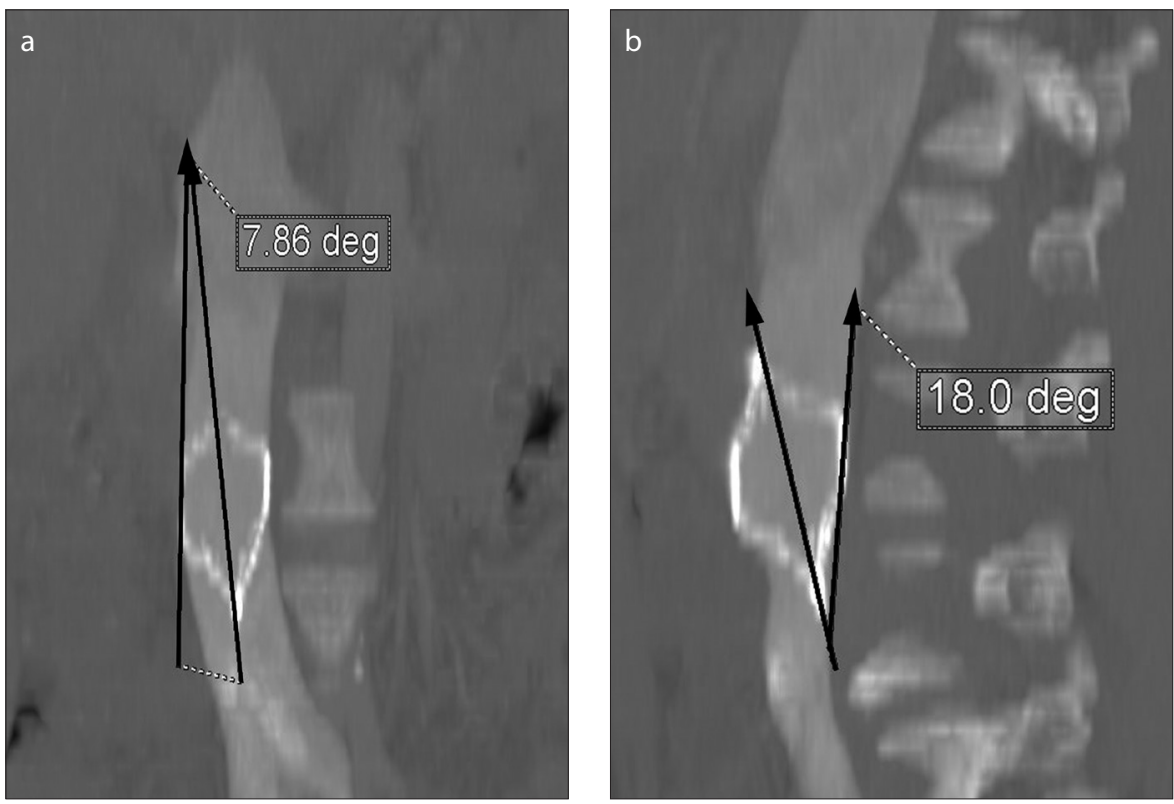

Figure 2. a, b. Significant filter tilt. Reconstructed coronal CT image (a) shows the axis of the IVC filter with a tilt angle of $7.86^{\circ}$. Reconstructed sagittal CT image (b) shows the axis of the IVC filter with a tilt angle of $18^{\circ}$. Arrows in (a) and (b) indicate angle created by the axis of the filter with respect to the IVC.

as the calf veins, as well as the proximal iliofemoral veins. In all patients, recanalization procedures (such as thrombectomy or catheter-directed thrombolysis) and IVC filter insertion for the prevention of PE were performed sequentially through a single popliteal vein access site. Aspiration thrombectomy $(n=21)$ and catheter-directed thrombolysis $(n=16)$ were performed to recanalize the occluded lower extremity veins immediately after IVC filter deployment. For catheter-directed thrombolysis, urokinase was infused continuously for several hours (range, 1.5-4 hrs, mean, 3.21 \pm 0.95 hrs) at $100000 \mathrm{IU} / \mathrm{h}$. The median amount of infused urokinase was $330000 \mathrm{IU}$ (range, 140 000-540 $000 \mathrm{IU}$ ). The mean duration of aspiration thrombectomy was $11.48 \pm 5.16$ min (range, 5-22 min). Adjuvant endovascular treatment, including venous stent or balloon angioplasty in cases of flow-limiting venous pathology, was performed in 15 patients. The mean amount of contrast media used during IVC filter insertion and PEVI was $114.76 \pm 23.58 \mathrm{~mL}$ (range, $80-150 \mathrm{~mL}$ ). There were no major complications during or after the procedure. Either follow-up CT venography $(n=17)$ or Doppler ultrasonography $(n=4)$ was performed in all patients. Rethrombosis of treated segments were observed in 6 of 21 patients (28.6\%) during the follow-up period.

In all patients $(n=21)$, OptEase filters were successfully deployed in the infra-renal IVC through the ipsilateral popliteal vein access site. Balloon angioplasty of the iliac vein was needed in one patient with severe iliac vein stenosis prior to filter deployment. Follow-up CT venography data were obtained in 17 patients within two weeks of the procedure. The degree of filter tilt in each patient is listed in the Table. The measured mean filter tilt was $7.14^{\circ} \pm 4.48^{\circ}$ in the coronal plane and $8.77^{\circ} \pm 5.49^{\circ}$ in the sagittal plane. Although the sagittal filter tilt was larger than the coronal filter tilt, this difference was not statistically significant $(P=$ 0.238 ). Considerable filter tilt, defined as tilt $\geq 15^{\circ}$, was observed in three patients, and the maximal filter tilt was $18^{\circ}$ (Fig. 2). In 13 patients who underwent IVC filter insertion via contralateral femoral access, the measured mean filter tilt was $6.03^{\circ} \pm 3.48^{\circ}$ in the coronal plane and $6.99^{\circ} \pm 1.87^{\circ}$ in the sagittal plane. Although the mean filter tilt in the contralateral femoral access group was smaller than the ipsilateral popliteal access group, this difference was not statistically significant in the coronal $(P=0.464)$ or sagittal $(P=0.229)$ planes.

Filter retrieval was attempted in 17 of 21 patients. The indication for permanent filter placement was persistent DVT in three patients and patient refusal of the retrieval procedure in one patient. In 16 patients, the OptEase filter was successfully removed after a mean filter dwell time of 20.13 days. The longest filter dwell time was 37 days. In one patient, the filter could not be removed because the caudal hook of the filter was embedded into the IVC wall: the sagittal tilt of the filter in this patient was $14.3^{\circ}$.

\section{Discussion}

Generally, IVC filter insertion and PEVI require two separate procedures performed on different days because of the need for different access sites. Popliteal vein IVC filter insertion is an efficient procedure that can 


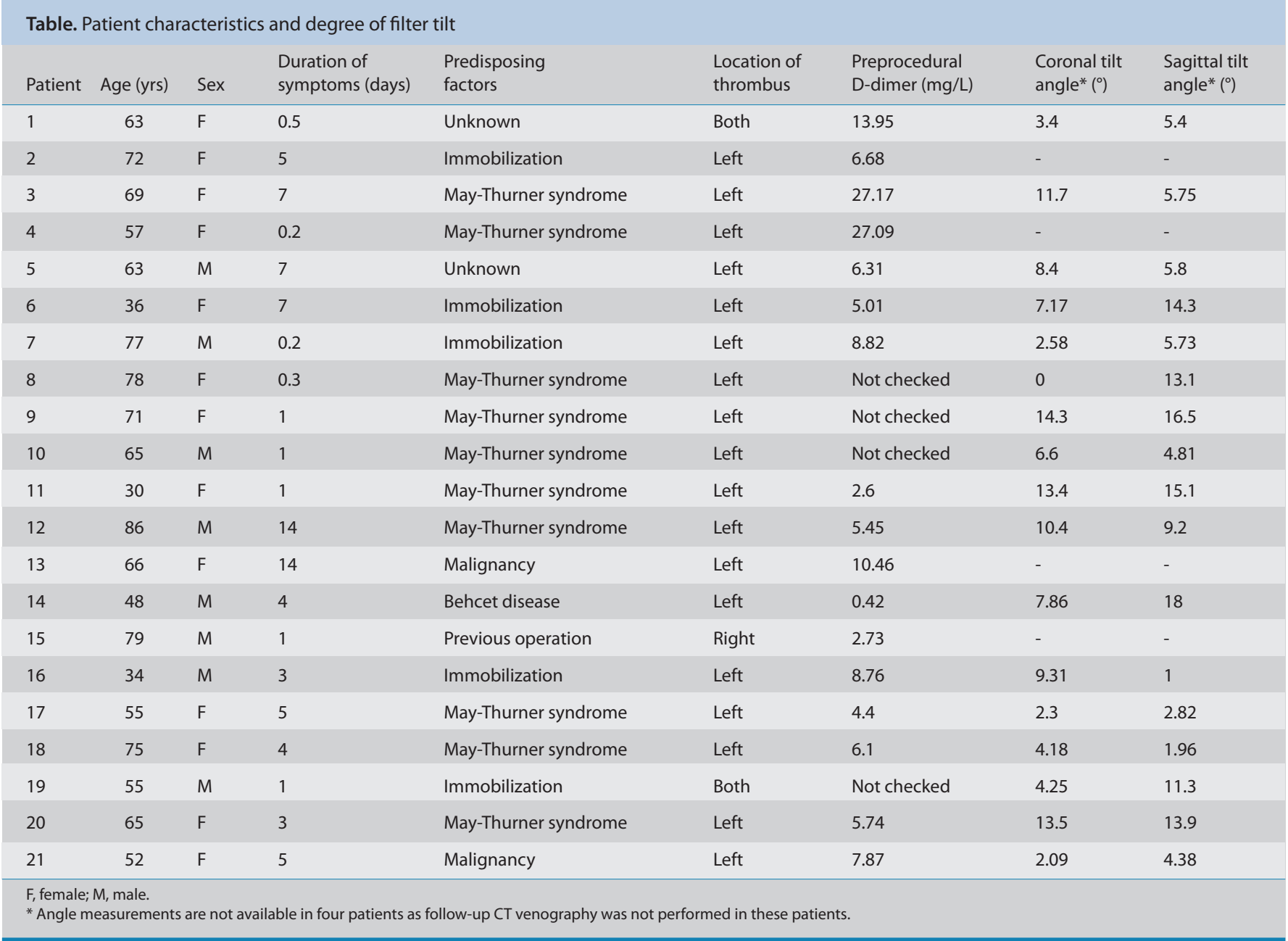

be performed in conjunction with PEVI in a single session through a single access site. In this study, the filter was successfully placed in all patients without procedural or postprocedure complications. Additionally, popliteal vein IVC filter insertion was associated with a low occurrence of significant filter tilt.

Venous thromboembolism, manifesting as DVT and PE, is a potentially lethal disorder that affects 1 in 1000 individuals annually (5). PE represents a serious cause of morbidity and mortality, particularly in hospitalized patients, and is estimated to cause over 200000 deaths annually in the United States (6).

PEVI has been shown to improve patients' quality of life compared to anticoagulation alone, and it has replaced systemic thrombolysis because of its higher recanalization rate and fewer bleeding-related complications $(7,8)$. PEVI frequently involves the instrumentation of extensive amounts of fresh thrombus, which may lead to PE. Mechanical stress applied to the clot by catheter manipulation, mechanical thrombectomy, stenting, and the accelerated lytic process in catheter-directed thrombolysis is hypothesized to increase the number and size of emboli resulting in symptomatic or silent PE (9). Placement of a retrievable IVC filter during thrombolytic therapy can prevent silent and symptomatic PE during PEVI (3). The incidence of iatrogenic PE during PEVI has been reported to be as high as $33 \%-45 \%(3,10)$. An eight-fold increase in iatrogenic symptomatic PE in those not receiving a filter was noted by Sharifi et al. (11).

In general, the IVC filter is inserted several days prior to thrombolytic therapy. This is done because if these two procedures are performed on the same day, bleeding may occur at the access site of filter insertion, which is typically the femoral or internal jugular vein. However, in this study, all filters were placed using the same popliteal access site used for PEVI, thereby avoiding the need for patient repositioning. Using the same access site allows filter placement and PEVI to be performed sequentially in a single procedure. The OptEase filter has a $6 \mathrm{~F}$ system with a $90 \mathrm{~cm}$ long introducer sheath, allowing insertion of the filter from the popliteal vein into the infrarenal IVC. In our experience, all OptEase filter insertions through the popliteal vein access site were successful. Furthermore, a single-session procedure can decrease the length of time that the filter is in place. The incidence of complications associated with IVC filters is time dependent. Recent data from a systematic review of 37 studies confirmed the increased rate of complications when filters are left in place for $\geq 30$ days and reported a retrieval rate of approximately $34 \%$ (12). The risks of unretrieved filters include recurrent DVT, vena cava thrombosis, organ penetration, and mechanical filter complications such as migration or fracture.

The transpopliteal insertion of an IVC filter carries the potential risk of filter tilt. Excessive tilt of the filter during placement may increase the risk of ineffective thrombus filtering, perifilter thrombosis, and retrieval failure and may also promote vascular remodeling (13-19). Retrieving filters 
that are tilted requires a longer procedure, increases radiation exposure, and may increase the overall risk of complications. In our study, significant filter tilt occurred in three $(14.3 \%)$ patients, which is less than or equal to the tilt rates reported in other published studies (20-22). With these relatively low tilt rates, our rate of filter retrieval was $76.2 \%$, which is higher than that reported in other published studies $(12,23-25)$. Filter retrieval was successful in 16 of 17 patients (94.1\%) in whom filter retrieval was attempted. In one patient, the filter could not be removed because the caudal hook of the filter was embedded into the IVC wall.

There are some limitations in this study. First, the limited number of patients from a single institution may limit generalization. Second, filter placement through the ipsilateral popliteal vein has a potential risk of iatrogenic PE because the filter delivery system may be passed through the sites of thrombus formation. However, the relatively small $6 \mathrm{~F}$ system of the OptEase filter, along with gentle manipulation during filter insertion, can reduce the risk of iatrogenic PE. In this study, none of the patients experienced symptomatic PE. Sharifi et al. (11) also showed that filter placement through the popliteal vein and extensive thrombus was not associated with a higher complication rate than filters placed in thrombus-free sites. Although we feel that this procedure is associated with a relatively low risk for iatrogenic $P E$, future studies using postprocedural multidetector $\mathrm{CT}$ of the pulmonary arteries or ventilation/perfusion scintigraphy are required to further assess the risk of iatrogenic PE.

In conclusion, our study suggests that popliteal vein IVC filter insertion is an efficient procedure that can be performed in conjunction with PEVI in a single session through a single access site. This method is also associated with a low incidence of significant filter tilt.
Conflict of interest disclosure

The authors declared no conflicts of interest.

\section{References}

1. Chung J, Owen RJ. Using inferior vena cava filters to prevent pulmonary embolism. Can Fam Physician 2008; 54:49-55.

2. Yamagami T, Nishimura T. Prophylactic implantation of inferior vena cava filter during endovascular therapies for deep venous thrombosis of the lower extremities. Ann Vasc Dis 2011; 4:19-23. [CrossRef]

3. Kolbel T, Alhadad A, Acosta S, Lindh M, Ivancev $\mathrm{K}$, Gottsater A. Thrombus embolization into IVC filters during catheter-directed thrombolysis for proximal deep venous thrombosis. J Endovasc Ther 2008; 15:605-613. [CrossRef]

4. Caplin DM, Nikolic B, Kalva SP, Ganguli S, Saad WE, Zuckerman DA; Society of Interventional Radiology Standards of Practice Committee. Quality improvement guidelines for the performance of inferior vena cava filter placement for the prevention of pulmonary embolism. J Vasc Interv Radiol 2011; 22:1499-1506. [CrossRef]

5. Deitelzweig SB, Johnson BH, Lin J, Schulman KL. Prevalence of clinical venous thromboembolism in the USA: current trends and future projections. Am J Hematol 2011; 86:217-220. [CrossRef]

6. Anderson FA, Zayaruzny M, Heit JA. Estimated annual numbers of US acute-care hospital patients at risk for venous thromboembolism. Am J Hematol 2007; 82:777-782. [CrossRef]

7. Comerota AJ, Throm RC, Mathias SD, Haughton S, Mewissen M. Catheter-directed thrombolysis for iliofemoral deep venous thrombosis improves health-related quality of life. J Vasc Surg 2000; 32:130-137. [CrossRef]

8. Hood DB, Alexander JQ. Endovascular management of iliofemoral venous occlusive disease. Surg Clin North Am 2004; 84:1381-1396, viii. [CrossRef]

9. Kolbel $\mathrm{T}$, Lindh $\mathrm{M}$, Holst J, et al. Extensive acute deep vein thrombosis of the iliocaval segment: midterm results of thrombolysis and stent placement. J Vasc Interv Radiol 2007; 18:243250. [CrossRef]

10. Thery C, Bauchart JJ, Lesenne M, et al. Predictive factors of effectiveness of streptokinase in deep venous thrombosis. Am J Cardiol 1992; 69:117-122. [CrossRef]

11. Sharifi M, Bay C, Skrocki L, Lawson D, Mazdeh S. Role of IVC filters in endovenous therapy for deep venous thrombosis: The FILTER-PEVI (filter implantation to lower thromboembolic risk in percutaneous endovenous intervention) trial. Cardiovasc Intervent Radiol 2012; 35:14081413. [CrossRef]
12. Angel LF, Tapson $\mathrm{V}$, Galgon RE, Restrepo Ml, Kaufman J. Systematic review of the use of retrievable inferior vena cava filters. J Vasc Interv Radiol 2011; 22:1522-1530. [CrossRef]

13. Singer MA, Wang SL. Modeling blood flow in a tilted inferior vena cava filter: does tilt adversely affect hemodynamics? J Vasc Interv Radiol 2011; 22:229-235. [CrossRef]

14. Grassi CJ. Inferior vena caval filters: analysis of five currently available devices. AJR Am J Roentgenol 1991; 156:813-821. [CrossRef]

15. Iliescu B, Haskal Z. Advanced techniques for removal of retrievable inferior vena cava filters. Cardiovasc Intervent Radiol 2012; 35:741-750. [CrossRef]

16. Marquess JS, Burke CT, Beecham AH, et al. Factors associated with failed retrieval of the Gunther Tulip inferior vena cava filter. J Vasc Interv Radiol 2008; 19:1321-1327. [CrossRef]

17. Geisbusch P, Benenati J, Pena C, et al. Retrievable inferior vena cava filters: factors that affect retrieval success. Cardiovasc Intervent Radiol 2012; 35:1059-1065. [CrossRef]

18. Stavropoulos SW, Dixon RG, Burke CT, et al. Embedded inferior vena cava filter removal: use of endobronchial forceps. J Vasc Interv Radiol 2008; 19:1297-1301. [CrossRef]

19. Kuo WT, Cupp JS, Louie JD, et al. Complex retrieval of embedded IVC filters: alternative techniques and histologic tissue analysis. Cardiovasc Intervent Radiol 2012; 35:588-597. [CrossRef]

20. Dinglasan LA, Oh JC, Schmitt JE, Trerotola SO, Shlansky-Goldberg RD, Stavropoulos SW. Complicated inferior vena cava filter retrievals: associated factors identified at preretrieval CT. Radiology 2013; 266:347-354. [CrossRef]

21. Sangwaiya MJ, Marentis TC, Walker TG, Stecker M, Wicky ST, Kalva SP. Safety and effectiveness of the Celect inferior vena cava filter: preliminary results. JVasc Interv Radiol 2009; 20:11881192. [CrossRef]

22. McConville RM, Kennedy PT, Collins AJ, Ellis PK. Failed retrieval of an inferior vena cava filter during pregnancy because of filter tilt: report of two cases. Cardiovasc Interv Radiol 2009; 32:174-177. [CrossRef]

23. Ray CE Jr, Mitchell E, Zipser S, Kao EY, Brown CF, Moneta GL. Outcomes with retrievable inferior vena cava filters: a multicenter study. J Vasc Interv Radiol 2006; 17:1595-1604. [CrossRef]

24. Mission JF, Kerlan RK Jr, Tan JH, Fang MC. Rates and predictors of plans for inferior vena cava filter retrieval in hospitalized patients. J Gen Intern Med 2010; 25:321-325. [CrossRef]

25. Minocha J, Idakoji I, Riaz A, et al. Improving inferior vena cava filter retrieval rates: impact of a dedicated inferior vena cava filter clinic. J Vasc Interv Radiol 2010; 21:1847-1851. [CrossRef] 